\title{
Die gesellschaftliche Einbettung autonomer Fahrzeuge am Beispiel Bad Birnbach
}

\author{
Alexandra Appel, Jürgen Rauh, Maximilian Graßl \\ und Sebastian Rauch
}

Innovationen und Neuerungen stehen in permanenter Wechselwirkung mit gesellschaftlichen Akteuren und Kontexten. Dazu gehören u. a. organisatorische oder Unternehmenskontexte, rechtliche Rahmenwerke, Nutzer und Nicht-Nutzer, Diskurse in der breiteren Öffentlichkeit sowie transnationale Kontexte. Diese Kontexte tragen dazu bei, Neuerungen und Innovationen gesellschaftlich zu legitimieren oder zu blockieren. Am Fallbeispiel des Pilotprojekts zu autonom fahrenden Kleinbussen im ÖPNV in Bad Birnbach werden relevante Akteure und Prozesse für die erfolgreiche Umsetzung in zwei Schritten analysiert. Im ersten Schritt wird das Gesamtnetzwerk aller involvierten Akteure erstellt und hinsichtlich wichtiger Mediatoren im Netzwerk untersucht. Im zweiten Schritt werden die einzelnen Projektphasen mit Hilfe der zuvor genannten fünf Dimensionen gesellschaftlicher Einbettungsprozesse analysiert. Es kann gezeigt werden, dass Einbettungsprozesse in die unterschiedlichen Dimensionen zeitversetzt erfolgen und teilweise aufeinander aufbauen. Schlüsselrollen nehmen dabei Akteure ein, die Erfahrungen in oder Zugang zu unterschiedlichen gesellschaftlichen Teilbereichen haben oder die Handlungsmacht anderer Akteure durch Legitimation und Befürwortung begünstigen können. Methodische Grundlage bilden Leitfadeninterviews und soziale Netzwerkanalyseverfahren.

A. Appel $(\bowtie) \cdot$ J. Rauh $\cdot$ M. Graß1 $\cdot$ S. Rauch Julius-Maximilians-Universität Würzburg, Würzburg, Deutschland E-Mail: alexandra.appel@uni-wuerzburg.de; juergen.rauh@uni-wuerzburg.de; maximilian. grass1@stud-mail.uni-wuerzburg.de; sebastian.rauch@uni-wuerzburg.de 


\subsection{Einleitung}

Autonome und fahrerlose Fahrzeuge erhalten zunehmend Aufmerksamkeit im öffentlichen Diskurs über den möglichen Wandel bestehender Verkehrs- und Mobilitätsysteme und -technologien. Dabei kann bislang noch nicht von autonomen Fahrzeugen auf öffentlichen Straßen gesprochen werden. Zu unzuverlässig sind die Technologien noch in unvorhersehbaren Situationen, rechtliche Rahmenwerke sind noch nicht angepasst und ethische und moralische Grundlagen müssen noch verhandelt werden. Die meisten der derzeit vermeintlich autonom fahrenden Fahrzeuge sind laut Kategorisierung der Society of Automotive Engineers International (SAE, deutsch: Verband der Automobilingenieure) nur (teil-)automatisiert und können zwar eigenständig bestimmte Manöver durchführen, stehen aber permanent unter Überwachung durch einen menschlichen Operator, also einem Bereitschaftsfahrer, der jederzeit eingreifen kann (Kap. 5, Kolb et al.). Das gilt auch für das Pilotprojekt in Bad Birnbach, wo seit Oktober 2017 die „erste autonome Buslinie Deutschlands im öffentlichen Straßenraum“ getestet wird (DB 2018).

Im öffentlichen Diskurs wird trotzdem wiederholt von autonomen oder fahrerlosen Fahrzeugen gesprochen. Das heißt, auch wenn die einzelnen Fahrzeuge im engeren Sinne nicht autonom verkehren, tragen Projekte wie in Bad Birnbach dazu bei, technologische Neuerungen im Bereich autonomer und (teil-)automatisierter Fahrzeugsysteme gesellschaftlich auszuhandeln, zu legitimieren und eine Verbreitung der Technologien zu befördern (oder zu verhindern). Diese Prozesse werden im vorliegenden Beitrag als ,societal embedding“ bzw. gesellschaftliche Einbettungsprozesse konzeptualisiert (z. B. Deuten et al. 1997, S. 131; Geels und Verhees 2011; Kanger et al. 2018).

Grundannahme ist, dass soziale Akteure (individuell und kollektiv) sowie ihre Interaktionen und die daraus entstehenden Netzwerke von grundlegender Bedeutung für die gesellschaftliche Akzeptanz und erfolgreiche Einführung von (technologischen) Neuerungen und Innovationen sind. Pilotprojekte, wie das in Bad Birnbach, können als anfängliche Diffusionsprozesse einer Technologie und eines Produkts (Kanger et al. 2018) angesehen werden, deren zukünftige Ausgestaltung allerdings noch keineswegs klar ist. Vielmehr handelt es sich bei dem automatisierten Kleinbus EZ10 (EasyMile 2018; Kap. 2, BarillèreScholz et al.) um eine Fahrzeuginnovation, deren Potenziale - auch im Hinblick auf die gesellschaftliche Legitimation der Technologie und des Produkts - im Rahmen einer Testphase unter möglichst realen Bedingungen analysiert werden können.

Solche technologischen Neuerungen stehen in Wechselwirkung mit den sie umgebenden sozialen, gesellschaftlichen, politischen oder wirtschaftlichen Systemen, die die Verbreitung der Technologie steuern (z. B. rechtliche Rahmenwerke, Mobilitätsroutinen, Verkehrspolitik). Allerdings beeinflussen die Anforderungen der Technologie auch die sie umgebenden räumlichen und gesellschaftlichen Kontexte und Strukturen (z. B. Infrastrukturen, rechtliche Neuregelungen, Alltagspraktiken). Wegen dieser Wechselwirkungen können gesellschaftliche Einbettungsprozesse auf unterschiedlichen Ebenen auch als Co-Konstruktion von Technologie und gesellschaftlichem Kontext verstanden werden. Co-Konstruktion ist ,,[...] a process of societal embedding, in which technologies find 
their place in wider societal domains, which include immediate user contexts, cultural meanings, policies, and infrastructures" (Kanger et al. 2018, S. 1). Diese Prozesse sind nicht gerichtet, sondern verlaufen schleifenartig und haben Rückkopplungen, die sowohl auf die Technologie als auch auf den gesellschaftlichen Kontext zurückwirken. Auch wenn im Fall von Bad Birnbach also noch nicht von einer weitreichenden Disruption bestehender Systeme gesprochen werden kann (Kap. 1, Derer und Geis), tragen das Projekt und die Präsenz in der Öffentlichkeit dazu bei, automatisierte und autonome Fahrzeugtechnologien gesellschaftlich einzubetten.

Ziele des vorliegenden Beitrags sind die Identifikation und Analyse von Akteuren, Netzwerken und Prozessen für die Umsetzung und Legitimation (teil-)automatisierter Fahrzeugsysteme durch gesellschaftliche Einbettungsprozesse. Das ist nicht nur für das Fallbeispiel Bad Birnbach interessant, sondern für Technologieinnovationen und ihre gesellschaftliche Legitimation/Einbettung allgemein. Zudem sind diese Fragestellungen im Rahmen des vorliegenden Projekts von besonderer Bedeutung, da es sich bislang deutschlandweit um das erste Projekt eines automatisierten Systems im öffentlichen Personennahverkehr (ÖPNV) auf öffentlichen Straßen und im ländlichen Raum handelt. Kleine Kommunen werden bislang weitestgehend ausgeblendet in Diskussionen um Mobilitätswandel. Daraus können auch Rückschlüsse auf die Anforderungen einer Region oder Kommune zur Einführung solcher Art Projekte gezogen werden.

Im Folgenden werden zunächst die theoretischen Grundlagen zu Prozessen der gesellschaftlichen Einbettung und das methodische Vorgehen vorgestellt. Methodische Grundlagen des vorliegenden Beitrags bilden qualitative Interviews, inhaltsanalytische Auswertungsverfahren sowie qualitative und quantitative soziale Netzwerkanalysen. Auf dieser Grundlage werden zuerst die eher statische Netzwerkarchitektur des Gesamtnetzwerks aller relevanten Akteure für die Umsetzung rekonstruiert und Mediatoren im Netzwerk identifiziert. Im nächsten Schritt werden drei charakteristische Projektphasen definiert und die Dynamiken in der sich ändernden Netzwerkkonfiguration nachvollzogen.

\subsection{Gesellschaftliche Einbettung}

Das Konzept Embeddedness stammt aus der Soziologie (Polanyi 1957; Granovetter 1985) und beschreibt die Annahme, dass wirtschaftliche Aktivitäten eingebettet sind in Systeme aus sozialen und gesellschaftlichen Beziehungen. Diese Strukturen sozialer Beziehungen bestimmen die Organisation, Strategien und Handlungen von (kollektiven und individuellen) wirtschaftlichen Akteuren mit (Pike et al. 2000). Embeddedness kann als Zustand oder als Prozess konzeptualisiert werden. Vor dem Hintergrund der Forschung zu sozio-technologischem Wandel und Systemtransformationen haben Deuten et al. (1997) das dynamische Konzept societal embedding (gesellschaftliche Einbettungsprozesse) entwickelt. Grundgedanke des Konzepts ist, dass die Verbreitung von Neuerungen und Innovationen einer gesellschaftlichen Legitimation bedarf, die von weit mehr Faktoren als der Akzeptanz durch Nutzer bestimmt wird. Vielmehr müssen weitreichende und 
vielschichtige gesellschaftliche Einbettungsprozesse in bestehenden und neuen gesellschaftlichen Bereichen stattfinden. Dazu gehören neben den häufig betrachteten Marktstrukturen und Nutzergruppen (z. B. Early Adopters/Erstanwender) auch rechtliche und institutionelle Rahmenwerke, zahlreiche Interaktionen und Prozesse sowie Akteure und Akteursgruppen aus Wirtschaft, Politik und Zivilgesellschaft: „Societal embedding of new products - that is, their integration in relevant industries and markets, their admissibility with regard to regulation and standards, and their acceptance by the public [...]“ (Deuten et al. 1997, S. 131).

Deuten et al. (1997, S. 132) definieren drei unterschiedliche Dimensionen gesellschaftlicher Einbettungsprozesse neuer Produkte oder Technologien, die für eine gesellschaftliche Legitimation und ggf. Integration notwendig sind: a) das Unternehmensumfeld (oder: Organisationsumfeld), in dem Technologien in relevante Branchen, Unternehmensstrukturen und Märkte integriert werden müssen, b) das Regelungsumfeld, in dem Technologien mit Vorschriften, Regeln und Standards übereinstimmen müssen, und c) die Gesellschaft im weiteren Sinne, in der Technologien von der breiteren Öffentlichkeit akzeptiert werden und mit bestehenden gesellschaftlichen Normen und Überzeugungen übereinstimmen müssen (Kanger et al. 2018, S. 3). Während sich das Unternehmensumfeld auf die wirtschaftliche Tragfähigkeit bezieht, beziehen sich Regelungsumfeld und Gesellschaft im weiteren Sinne auf die regulatorische und kulturelle Legitimität. An dieser Stelle soll zudem der Begriff Unternehmensumfeld im weiteren Sinne verstanden werden, nämlich im weitesten Sinne einer Unternehmung, die bspw. auch als Institution oder Gemeinde verstanden werden kann. Dabei ähneln sich die Aspekte der Einbettung vor allem dahingehend, dass jeder Unternehmung eine Form von Organisationsstruktur und Wirtschaftlichkeit zugrunde liegt. Im Folgenden wird also die Dimension Unternehmensumfeld mit Organisationsumfeld übersetzt und dadurch auch für politische und VerwaltungsEinrichtungen anwendbar gemacht.

Die oben genannten Einbettungsdimensionen wurden im Rahmen weiterführender Arbeiten ergänzt, sodass momentan von fünf Dimensionen gesellschaftlicher Einbettungsprozesse gesprochen werden kann. Dabei werden die vorher genannten um die folgenden zwei ergänzt: e) Einbettungsprozesse in das Nutzerumfeld, gekennzeichnet durch kulturell spezifische und räumlich differenzierte Routinen, Möglichkeiten, Praktiken oder Bedeutungszuschreibungen (Kanger et al. 2018; Mylan et al. 2018) und f) Einbettungsprozesse in ein transnationales Umfeld, da die Entstehung neuer Technologien, die das Potenzial haben, ganze sozio-technische Systeme zu verändern, auch Einfluss auf Entwicklungen an ganz anderen Orten oder internationale Standards haben können (Kanger et al. 2018, S. 3). Während das Nutzerumfeld oft im Rahmen von Akzeptanzuntersuchungen (Kap. 9, Rauh et al.; Kap. 6, Wintersberger et al.) Berücksichtigung findet, lässt sich als Beispiel für Einbettung in ein transnationales Umfeld die europäische Gesetzgebung (z. B. Wiener Übereinkommen über den Straßenverkehr) anführen.

Neue Technologien und ihre gesellschaftliche Adoption werden grundlegend geprägt von gesellschaftlichen Sinnzuschreibungen (Geels und Verhees 2011) und Diskursen über die jeweiligen Produkte und Technologien. So können selbst nachhaltige oder sinnvolle 
Produkte wieder vom Markt verschwinden, wenn die öffentlichen Diskurse negativ oder von Ängsten und Befürchtungen geprägt sind. Solche Diskurse wirken sich nicht nur auf die Wahrnehmungen und Meinungen von zivilgesellschaftlichen Akteuren aus, sondern auch auf potenzielle Geldgeber, oder rechtliche Rahmenwerke, die Innovationen befördern oder blockieren.

Zentral für das Konzept gesellschaftlicher Einbettungsprozesse ist die Reflexivität zwischen Technologie/Produkt und Kontext, die einen Aushandlungsprozess darstellt. Wiederholt wird also auf Co-Konstruktions-Prozesse zwischen Technologie und gesellschaftlichem Kontext verwiesen (z. B. Kanger et al. 2018). Produktinnovationen und ihre gesellschaftliche Einführung sind keineswegs linear oder stringent, sondern vielmehr gekennzeichnet durch eine Vielzahl von Anpassungsprozessen (z. B. Produktanpassungen, rechtliche Rahmenwerke, Infrastrukturen), Rückkopplungen (z. B. Innovationsprozesse) oder Interessenkonflikten unterschiedlicher Akteursgruppen (z. B. unternehmerische vs. ökologische Interessen), die nur begrenzt im Rahmen von Konzepten wie Marktstrukturen mit statischen Eigenschaften, Nutzergruppen mit definiertem Adoptionsverhalten oder Betrachtungen von Barrieren für die Anwendung, dargestellt werden können (Kanger et al. 2018). Auch wenn Aspekte wie Nutzeranforderungen oder Adoptionsverhalten nützliche Ansätze zur Produktverbesserung oder zur Einstufung einer Marktreife sein können, bleiben sie lückenhaft im Hinblick auf das Erklärungspotenzial der komplexen Dynamiken und Akteurskonstellationen, die zu gesellschaftlichen Legitimations- und Adoptionsprozessen von Neuerungen und Innovationen führen.

Die Bedeutung gesellschaftlicher Einbettungsprozesse und die daraus entstehende Legitimität für neue Produkte oder Technologien wurde vielfach in der Transitionsforschung vor dem Hintergrund neuer Technologien mit weitreichender gesellschaftlicher Relevanz, wie etwa neue Energiesysteme, angewendet. Dazu gehören Arbeiten z. B. zur historischen Perspektive auf die gesellschaftliche Legitimation nuklearer Energiequellen in den Niederlanden (Geels und Verhees 2011), in Bezug auf die Verbreitung erneuerbarer Energien in Deutschland (Jacobsson und Lauber 2006) oder in Bezug auf Niedrigenergiehäuser in Großbritannien (Lovell 2008). Aber auch in anderen Kontexten und Disziplinen wurde das dynamische Konzept angewendet. Mylan et al. (2018) beschreiben sog. Nischen-RegimeWechselwirkungen im Rahmen gesellschaftlicher Einbettungsprozesse bei Produktinnovationen im Milchsektor, Appel (2016) analysiert unterschiedliche Einbettungsdimensionen vor dem Hintergrund von Online-Lebensmitteleinzelhandel für Unternehmen, Märkte und Kunden und Coe und Lee (2013, S. 330) schreiben von einer ,deepening territorial embeddedness“ bei der Internationalisierung einer Supermarktkette in Süd-Korea.

Vor dem Hintergrund von Transformationsprozessen im sozio-technischen System Automobilität (Verbrennungsmotor und Elektroantrieb) erheben Kanger et al. (2018, S. 2) den Anspruch, die Ersten zu sein, die das Konzept societal embedding anwenden. Ihre Gegenüberstellung der historischen Entwicklungen der Antriebstechnologien in den USA und in den Niederlanden stellt gut nachvollziehbar die unterschiedlichen Diskurse über die jeweiligen Technologien und ihre Auswirkungen auf und Wechselwirkungen mit der gesellschaftlichen Einbettung dar. 
In dem vorliegenden Beitrag dient das Konzept als Analyserahmen zur Identifikation der im konkreten Fallbeispiel stattfindenden Prozesse und Einbettungsdimensionen. So können einerseits wichtige Dynamiken und Prozesse dargestellt, die jeweiligen gesellschaftlichen Einbettungsdimensionen und die Handlungsmacht der einzelnen Akteure rekonstruiert und andererseits Rückschlüsse auf die Relevanz der einzelnen Dynamiken, Beziehungen und Akteure für die Legitimation der neuen Technologie abgeleitet werden. Methodische Grundlage dafür bilden Leitfadeninterviews und soziale Netzwerkanalysen. Im Folgenden wird das methodische Vorgehen erläutert.

\subsection{Methoden - soziale Netzwerkanalyse und Leitfadeninterviews}

\subsubsection{Soziale Netzwerkanalyse}

Innerhalb der sozialen Netzwerkforschung können grundsätzlich zwei methodische Ansätze unterschieden werden: qualitative und quantitative. Zudem muss zwischen Methoden der Datenerhebung und -auswertung unterschieden werden. Qualitative Erhebungsund Auswertungsmethoden in der Netzwerkanalyse erlauben einen detaillierten Einblick in spezifische Beziehungen zwischen einzelnen Akteuren und sind in der visuellen Gestaltung der Ergebnisdarstellung offen. Daneben kann untersucht werden, wie und unter welchen Bedingungen einzelne Verbindungen zustande gekommen sind. Dabei haben sich Interviews als qualitative Erhebungsmethode ebenso bewährt, wie das Ermitteln spezifischer Beziehungsausprägungen mithilfe konzentrischer Kreise, die (räumliche, soziale oder kulturelle) Distanzen abbilden können (Hollstein 2006). Während qualitative Methoden spezifische Erklärungsansätze zur Interpretation bestimmter Phänomene liefern können, gelingt es mit standardisierten und quantitativen Vorgehensweisen besser, große Mengen an Akteuren und Verbindungen innerhalb eines Beziehungsgeflechts umfassend zu analysieren und visualisieren. Dabei können grundlegende Strukturen auf drei wesentlichen Analyseebenen mit entsprechenden statistischen Indikatoren untersucht werden. Während eine Charakterisierung einzelner Akteure zumeist über Zentralitätsmaße erfolgt (z. B. closeness centrality und betweeness centrality), dient die Dichte als Indikator der gesamten Netzwerkeinschätzung und ermöglicht somit unter anderem einen Vergleich unabhängiger Netzwerke. Als dritte Ebene kann das statistische Identifizieren einzelner Akteursgruppen herangezogen werden, was vornehmlich als Modularität bezeichnet wird (Jansen und Diaz-Bone 2014).

Auch wenn beide methodischen Richtungen in der Vergangenheit, teils auch heute noch üblicherweise getrennt in der Netzwerkforschung angewendet werden (Jack 2010; Schnegg 2010), existieren einige neuere Studien, die sich die Vorteile einer Verbindung zunutze machen (u. a. Czernek-Marszałek 2017). Um innerhalb der vorliegenden Forschungsergebnisse einen möglichst umfassenden Einblick in die Entstehung und Struktur des Akteur-Netzwerks zu erhalten, wird auch hier sowohl bei der Datenerhebung, als auch bei der -auswertung unter 
Verwendung einer Methoden-Triangulation bzw. eines mixed methods-Ansatzes (Hollstein 2014) ein multimethodisches Vorgehen gewählt. Eine solche Kombination wird unverzichtbar, wenn über Effekte und Strukturen hinaus festgestellt werden soll, welche Strategien Akteure innerhalb des Netzwerks verfolgen (Franke und Wald 2006).

\subsubsection{Erhebungs- und Auswertungsmethoden}

Methodische Grundlage bildeten vor allem leitfadengestützte Interviews mit Vertretern kommunaler (z. B. Kurverwaltung, Bürgermeister) und regionaler (z. B. Landratsamt Rottal-Inn, Regierung von Niederbayern) Institutionen und Unternehmen (z. B. TÜV Süd, Regionalbus Ostbayern - RBO). Hinsichtlich der Auswertungsmethoden wurden zum einen Techniken der qualitativen Inhaltsanalyse für ein detailliertes Verständnis der komplexen Prozesse und der Akteurskonstellationen im zeitlichen Verlauf angewendet. Zum anderen wurden qualitative und quantitative soziale Netzwerkanalysen, zur Darstellung der Akteurskonstellationen und der Identifikation von wichtigen Mediatoren und hybriden Akteuren, die Zugang zu unterschiedlichen gesellschaftlichen Teilbereichen (z. B. kommunale und landesweite Politik) im Netzwerk haben, durchgeführt.

Insgesamt konnten elf Interviews im Zeitraum von Februar bis September 2018 durchgeführt werden. Die Interviewpartner wurden entlang des Schneeballsystems ausgewählt. Das heißt, zentrale Akteure wurden nach weiteren wichtigen Akteuren und Personen, mit denen sie im Laufe des Projekts in Kontakt standen, gefragt. Die Interviewdauer lag zwischen einer und drei Stunde/n. Rückfragen konnten telefonisch oder per E-Mail geklärt werden. Zwei Interviews wurden telefonisch durchgeführt. Ein Interview fand als Gruppeninterview mit zwei Gesprächspartnern gleichzeitig statt. Alle Interviews konnten mit Zustimmung der Interviewpartner aufgezeichnet und im Nachgang transkribiert werden.

Nach Durchführung der Interviews folgten im Wesentlichen vier Phasen der Auswertung und Differenzierung der Daten. In einem ersten Schritt wurden die Interviews transkribiert. Auf Grundlage der Interviewtranskripte konnte in einem zweiten Schritt ein Gesamtnetzwerk an Akteuren rekonstruiert und Akteur-Matrizen (inklusive Informationen zur zeitlichen Dynamik und Relevanz der Akteure) angelegt werden, die den jeweiligen Interviewpartnern zur Bestätigung und Ergänzung zugesandt wurden. In einem dritten Schritt konnte eine detaillierte Inhaltsanalyse durchgeführt werden. Auf Grundlage der Interviewtranskripte, des rekonstruierten Akteur-Netzwerks und der Akteur-Matrizen mit den zeitlich-dynamischen Informationen, konnten einerseits die charakteristischen Projekt- und Prozessphasen und andererseits die jeweils relevanten Akteurskonstellationen und ihre Charakteristika sowie Bedeutung für die Dimensionen der gesellschaftlichen Einbettung abgeleitet werden. In einem vierten Schritt wurde auf Basis der Interviewtranskripte, der Akteur-Matrizen und der identifizierten Phasen eine Datenbank angelegt, auf deren Grundlage die empirischen Ergebnisse der qualitativ ausgewerteten Netzwerkanalyse, mittels quantitativer Auswertungsmethoden der sozialen Netzwerkanalyse, überprüft werden konnten. 
Unter Berücksichtigung der Aussagen befragter Akteure kann des Weiteren eine Auswertung bestimmter Beziehungsstrukturen und Akteurskonstellationen zu unterschiedlichen Zeitabschnitten betrachtet werden. Eine solche dynamische Netzwerkanalyse gilt als eine der größten Herausforderungen der Netzwerkforschung und ermöglicht weiterführende Aussagen sowohl über die Netzwerkentstehung und -veränderung als auch über die Orientierungen und Handlungsstrategien einzelner Akteure (Hollstein 2006).

\subsection{Gesellschaftliche Einbettung (teil-)automatisierter Fahrzeugsysteme}

Die Reaktionen auf das Pilotprojekt zur Einführung des ,,autonom fahrenden Kleinbusses in Bad Birnbach" sind gekennzeichnet durch ein hohes Maß an Unterstützung über alle Akteursgruppen hinweg. Es gab keinerlei Widerstände und Proteste gegen die Einführung. Auch die Anwohner und Nutzer zeichnen sich grundsätzlich durch Offenheit gegenüber neuen Mobilitätsmodellen und -technologien aus - auch wenn individuelle Zweifel an Finanzierbarkeit und Zukunftsfähigkeit des derzeitigen Shuttle-Busses durchaus bestehen können (Kap. 9, Rauh et al.). Trotzdem haben während des gesamten Einführungsprozesses diverse Rückkopplungs- und Anpassungsprozesse zwischen den unterschiedlichen gesellschaftlichen Dimensionen stattgefunden. Besonders hervorzuheben sind die Wechselwirkungen (Co-Konstruktion) während der Vorbereitungsphase, in die auch der Zulassungsprozess des Fahrzeugs fällt. Von einem linearen Prozess kann jedenfalls keine Rede sein.

In den folgenden Abbildungen werden qualitative (Position und Farbe) und quantitative (Größe) Merkmale der Akteure kreisförmig dargestellt. Die Größe beschreibt die Zentralität der Akteure im Netzwerk gemessen an seiner betweenness centrality oder Zwischenzentralität, also die Anzahl der kürzesten Verbindungen, die durch diesen Knoten/Akteur verlaufen. Zentralität als netzwerkanalytisches Konzept dient als Indikator für die Rolle eines einzelnen Akteurs innerhalb einer Akteurskonstellation (Jansen 2006). Zwischenzentralität ist vor allem ein Maß für die mediale Rolle eines Akteurs, also über wie viele direkte und indirekte Kontakte er verfügt und so auch zwischen unterschiedlichen Teilbereichen vermitteln und kommunizieren kann. Je größer ein Akteur demnach visualisiert ist, desto höher ist seine Zwischenzentralität im Gesamtnetzwerk (Abb. 8.1) und während der einzelnen Projektphasen (Abb. 8.2).

Allerdings ist diese visualisierte Größe der Akteure im vorliegenden Netzwerk mit Vorsicht zu betrachten, da nur ein Teil der genannten und dargestellten Akteure persönlich befragt werden konnte. Es ist davon auszugehen, dass die befragten Akteure daher hinsichtlich der Verbindungen im Netzwerk überrepräsentiert sind. Gerade in der Darstellung des Gesamtnetzwerkes ist die Aussagekraft der Größe der Akteure teilweise begrenzt, in den Darstellungen der zeitlichen Abfolge hingegen kann über die sich verändernde Größe der einzelnen Akteure gut visualisiert werden, wie sich die Relevanz der Akteure zu unterschiedlichen Projektphasen verändert hat. 
Die Position der Akteure innerhalb des Netzwerks gibt Auskunft über die Zuordnung der Akteure zu einer der räumlichen und verwaltungsrechtlichen Ebenen Kommune, Region, Land (Freistaat Bayern) oder Bund (Deutschland). Die außerhalb dieser konzentrischen Kreise angeordneten Akteure sind als transnationale Akteure zu verstehen, die über Ländergrenzen hinweg agieren. Die Farbe zeigt die Zuordnung der Akteure zu einem der gesellschaftlichen Teilbereiche Wirtschaft (rot), Verwaltung (grün), Politik (blau), Zivilgesellschaft (gelb) oder Presse (orange) an.

Die Verbindungslinien stellen rein qualitative Informationen dar, und enthalten keine Informationen über Häufigkeiten oder Relevanzen von Interaktionen. Auch die Farben der Verbindungslinien enthalten keine statistischen Informationen, sondern dienen auf qualitativer Ebene der leichteren Erkennbarkeit von Akteurs-Clustern, die dem gleichen gesellschaftlichen Teilbereich angehören, bzw. daran ist zu erkennen, zwischen welchen Institutionen der Austausch über gesellschaftliche Teilbereiche hinaus stattgefunden hat.

\subsubsection{Legitimation des Projekts durch Mediatoren und hybride Akteure im Gesamtnetzwerk}

In Abb. 8.1 wird das Gesamtnetzwerk, also die Gesamtheit aller Akteure und Interaktionen, die aus den Interviewtranskripten und den Akteurs-Matrizen abgeleitet wurden, dargestellt. Abgebildet ist der aus den Interviews abgeleitete Gesamtzeitraum (Anfang des Jahres 2015 bis Dezember 2018) von dem ersten eher informellen Kontakt zwischen Vertretern der Gemeinde Bad Birnbach und Vertretern der Deutschen Bahn (DB). Als besonders relevant werden die Akteure angesehen, die zwischen unterschiedlichen Teilbereichen der Gesellschaft kommunizieren und vermitteln können, da sie entweder Zugang zu oder Erfahrungen in mehreren Teilbereichen haben und so auch über die entsprechenden Kontakte und Wissen über milieuspezifische Codes verfügen. Diese Akteure tragen dazu bei, die Legitimation einer Technologie in anderen Teilbereichen der Gesellschaft voranzutreiben und können verlängerter Arm der Handlungsmacht der Akteure sein, die ohne Kontakte und Beziehungen zu Akteuren aus anderen gesellschaftlichen Teilbereichen eingeschränkt wären. Gesellschaftliche Teilbereiche können sowohl vertikal, als auch horizontal gegliedert sein. Beispielsweise können Wirtschaft und Ökologie ebenso als unterschiedliche Teilbereiche aufgefasst werden, wie kommunale Politik und Bundespolitik.

Insgesamt ließen sich aus den Datengrundlagen 60 (kollektive und individuelle) Akteure und 106 Verbindungen ableiten. Während die Anzahl der Akteure im Netzwerk als repräsentativ einzustufen ist, da die genannten Akteure in mehreren Interviews bestätigt werden konnten, ist davon auszugehen, dass die Anzahl der einzelnen Interaktionen und Beziehungen weniger zuverlässig und repräsentativ im statistischen Sinne abgefragt werden konnten. Grund dafür ist auch, dass nicht jede genannte Person im Netzwerk im Rahmen eines Leitfadeninterviews befragt wurde. Teilweise stellen die Interviewpartner daher Repräsentanten ihrer Unternehmen oder Institutionen dar. In den Interviews wurden demnach nicht nur egobasierte Netzwerke abgefragt und erhoben, sondern auch Strukturen des Gesamtnetzwerks. Es kann zuverlässig dargestellt werden, welche Beziehungen zwischen 
einzelnen Institutionen und Einzelakteuren bestehen, die Intensität ist wegen der fehlenden Daten aber nur bedingt darstellbar.

Außerdem ist darauf hinzuweisen, dass die Akteure der Begleitforschung, die insbesondere ab Februar 2017 immer wieder mit unterschiedlichen Akteuren und dem automatisierten Bus interagieren, nicht im Netzwerk dargestellt sind. Darauf wurde verzichtet, da sie eher eine beobachtende Funktion übernehmen und für die Umsetzung des Projekts über keine spezifische Relevanz verfügen. Trotzdem muss berücksichtigt werden, dass die publizierten Ergebnisse der Begleitforschung zukünftig - gewollt oder nicht gewollt - zur Legitimation solcher oder ähnlicher Projekte herangezogen werden können. Auch ist davon auszugehen, dass die Interaktionen der Akteure mit den Vertretern der Begleitforschung zu Wechselwirkungen und Co-Konstruktionen führen können bzw. z. T. sogar darauf abzielen, dass das Produkt beispielsweise nutzerfreundlicher wird. Diese Aspekte werden in diesem Beitrag jedoch nicht berücksichtigt, da im Mittelpunkt die Akteure und Prozesse, die zur Umsetzung des Projekts führten, stehen.

Für die bessere Übersichtlichkeit und die Darstellung im vorliegenden Format wurden die Akteure zu Akteursgruppen aggregiert, sodass in dem aggregierten Netzwerk noch 39 Akteure bei gleicher Anzahl von Verbindungen (109) vorliegen. Aggregiert wurden Akteure, die gemeinsam in einer/m Institution/Unternehmen agieren. Nicht aggregiert wurden Akteure, denen eine zentrale, hybride Rolle im Netzwerk zugewiesen wurde. Personen mit repräsentativen Funktionen wurden ebenfalls nicht aggregiert, da ihnen eine besondere Rolle bei der Interaktion mit anderen Teilbereichen des Netzwerks zukommt.

Das soziale Netzwerk zur Umsetzung des Pilotprojekts in Bad Birnbach ist gekennzeichnet durch eine hohe Dichte an Akteuren und Verbindungen auf kommunaler, regionaler und Landes-Ebene (Abb. 8.1). ${ }^{1}$ Eine zentrale Rolle nimmt der Leiter der Kurverwaltung von Bad Birnbach (KurVW BaBi) ein. An dieser Stelle laufen die meisten Verbindungen, auch über die räumlichen Ebenen hinweg zusammen. Zudem bestehen von hier aus Kontakte zu allen gesellschaftlichen Teilbereichen (Zivilgesellschaft, Wirtschaft, Verwaltung, Politik und Presse). Dieser Akteur ist außerdem als hybrider Akteur anzusehen, da er sowohl im Verwaltungsapparat der Marktgemeinde (grün), als auch im Bereich der lokalen Presse- und Öffentlichkeitsarbeit (orange) aktiv ist. Dadurch ist dieser Akteur ein Mediator mit hoher Zwischenzentralität, der neben den institutionellen Akteuren auch in engem Kontakt mit zivilgesellschaftlichen Akteuren auf kommunaler und regionaler Ebene (z. B. Landwirte, Anwohner, Schulen, Regionalpresse) steht. Als Mediator zwischen kommunaler und regionaler Verwaltung und Politik und Zivilgesellschaft auf kommunaler und regionaler Ebene kann dieser Akteur öffentliche Diskurse und Wahrnehmungen des Pilotprojekts aktiv mitgestalten.

Eine weitere Instanz mit hoher Zentralität auf kommunaler Ebene stellt das Landratsamt Rottal-Inn (LRA Ro-I) dar. In der vorliegenden Abbildung ist die Zwischenzentralität dieses Akteurs eher gering dargestellt, da sowohl der Landrat (LR Ro-I) als auch der Leiter

\footnotetext{
${ }^{1}$ Nachfolgend werden alle Verweise auf im Netzwerk dargestellte Akteure im Text kursiv kenntlich gemacht.
} 


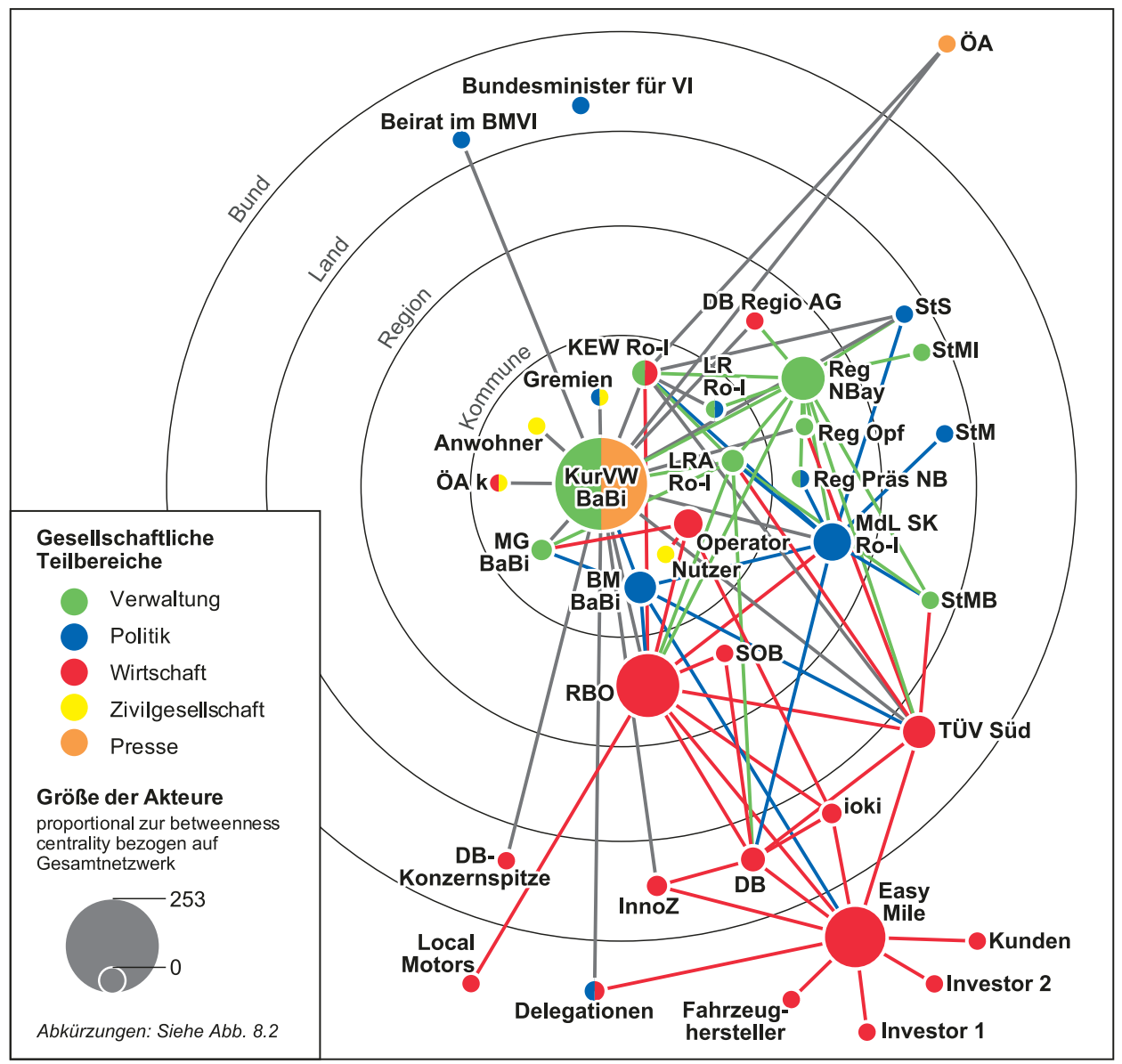

Abb. 8.1 Gesamtnetzwerk der Akteure für die Umsetzung des Pilotprojekts (Anfang 2015 Januar 2019). (Quelle: eigene Darstellung)

der Kreisentwicklung (KEW Ro-I), die beide wichtige Vertreter des Landratsamtes RottalInn sind, als eigene Knoten/Akteure dargestellt sind. Insgesamt verfügt die Institution mit ihren Vertretern aber über eine hohe Zwischenzentralität - vor allem in der Initial- und der Durchführungsphase (Abb. 8.2). Neben dem Landrat, der die Behörde nach außen repräsentiert, und den Mitarbeitern der Institution, die alle Operationen durchführen, kann der Leiter der Kreisentwicklung als zentral angesehen werden. Die Zentralität dieses Akteurs wird weniger durch die Vielzahl an Verbindungen definiert, als durch sein Selbstverständnis seiner Rolle als Leiter der Kreisentwicklung, in der er zwar für eine Verwaltung arbeitet, aber einen beruflichen Erfahrungshintergrund auch aus der freien Wirtschaft mitbringt: „Meine Vorgeschichte war Wirtschaftsförderer, ich war Regionalmanager, ich war Leader- 


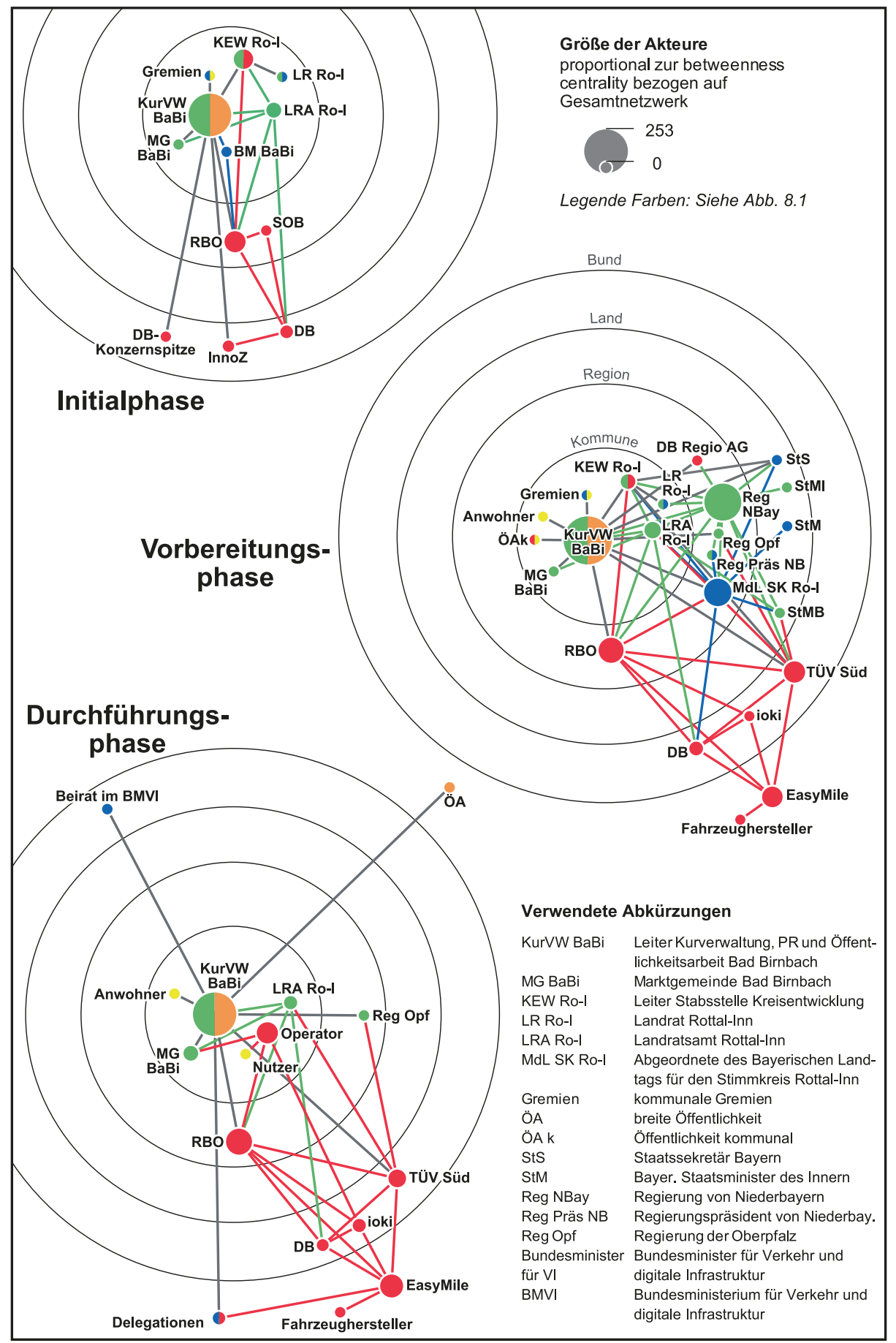

Abb. 8.2 Akteur-Netzwerke im zeitlichen Verlauf. (Quelle: eigene Darstellung) 
Manager und dann hat mich der neue Landrat in diesen Landkreis geholt und hat gesagt, ich soll da eine entsprechende Stelle für Regionalentwicklung aufbauen. [...] Ich sehe mich da nicht als Verwalter.“ (Interview Kreisentwicklung Rottal-Inn, 2018). Dieser Akteur hat demnach Erfahrungen und Kenntnisse in unterschiedlichen gesellschaftlichen Teilbereichen (Wirtschaft und Verwaltung) und kennt branchen- und milieuspezifische Handlungsweisen und Codes. Dadurch kann er als wichtiger Mediator zwischen diesen Teilbereichen angesehen werden.

Weniger zentral im Sinne der Zwischenzentralität erscheinen die Bürgermeister der Marktgemeinde Bad Birnbach ( $\mathrm{BM} \mathrm{BaBi}$ ), da sie in den Interviews vergleichsweise wenige Kontakte zu anderen Institutionen angeben. Allerdings unterstützten und legitimieren sie das Pilotprojekt, indem sie die untergeordneten Verwaltungseinheiten (z. B. die Bauverwaltung) mit entsprechenden Aufgaben betrauen.

Auf regionaler Ebene stellen vor allem die Landtagsabgeordnete für den Stimmkreis Rottal-Inn (MdL SK Ro-I) (Teilbereich Politik), das DB-Tochterunternehmen Regionalbus Ostbayern (RBO) (Teilbereich Wirtschaft) und die Regierung von Niederbayern (Reg NBay) (Teilbereich Verwaltung) wichtige Akteure mit hoher Zwischenzentralität dar. Die Landtagsabgeordnete stellt die wichtigste Mediatorin zwischen den unterschiedlichen politischen und verwaltungsrechtlichen Ebenen Kommune, Region und Land dar. Sie kommuniziert die Projektidee und ihre Befürwortung auf den der Kommune übergeordneten Verwaltungs- und politischen Ebenen, sodass die Finanzierung und die politische Unterstützung des Pilotprojekts auf den unterschiedlichen politischen Ebenen gewährleistet werden kann. „Mein Part war, nach oben zugehen [...] also sprich, ganz klar Minister zu begeistern und zu sagen, wir haben da was und das wollen wir jetzt in Bayern und speziell im Landkreis Rottal-Inn machen.“ (Interview Abgeordnete Stimmkreis Rottal-Inn, 2018).

Die RBO übernimmt ebenfalls eine zentrale Rolle einerseits als Geldgeber, andererseits als Projektkoordinator sowie als Akteur mit zahlreichen Verbindungen. Die RBO verfügt über Wissen hinsichtlich des Projektmanagements, Erfahrung im Bereich des straBenbasierten ÖPNV und der dazugehörigen verwaltungsrechtlichen Vorgehensweisen. „Eine Hauptrolle hatte die RBO. Damit haben wir auch einen sehr erfahrenen Betriebsleiter bekommen. Sie müssen sehen, da war ein Haufen junger Entwickler, die nichts anderes sehen als ihren Bus, da gibt's Strategen von der Bahn, und dazwischen drin waren dann wir [Vertreter von Bad Birnbach] und der Landkreis, und das hat natürlich schon sehr gut getan, jetzt wirklich mal einen expliziten Fachmann zu haben, der auch irgendwann mal gesagt hat: ,Leute, wie stellt ihr euch das denn vor? Das funktioniert in der Praxis nie!' Und ja, das war die Zutat, die gefehlt hat bei dem Ganzen, und seitdem die RBO da wirklich mit dabei ist, mit im Boot sitzt, seitdem hat das Ganze auch praxistaugliche Formen angenommen.“ (Interview Kurverwaltung Bad Birnbach, 2018). Die Rolle der RBO wurde ab 2017 durch die offizielle Ausgründung von ioki unterstützt.

Die Regierung von Niederbayern ist gerade hinsichtlich der verwaltungsrechtlichen Regelungen als Instanz mit hoher Zwischenzentralität anzusehen. Dort wird auch über die Verteilung öffentlicher finanzieller Ressourcen in Niederbayern entschieden. Das heißt, die grundsätzliche Befürwortung durch den Regierungspräsidenten von Niederbayern 
gegenüber höher gestellten politischen Institutionen (z. B. Bayerisches Staatsministerium des Innern - StMI, Bayerisches Staatsministerium für Wohnen, Bau und Verkehr - StMB) ermöglicht die Umsetzung des Projekts durch die Regelung der Finanzierbarkeit sowie der Zuständigkeiten im Zulassungsprozess, der keineswegs standardisiert ist, sondern durch Sonderzulassungen charakterisiert ist. „Also am Anfang, wenn wir irgendwo hingekommen sind, z. B. ins Innenministerium oder in eine Fachbehörde, dann war die Haltung eher die, ,Hoppla, warum kommt ihr zu mir? Ich bin nicht zuständig. Je länger das Projekt dann gelaufen ist und je mehr man dort auch erkannt hat, die Politik will es, die Öffentlichkeit will es, desto mehr hat sich hier auch die Stimmung gedreht, und plötzlich sind Strukturen entstanden [...].“ (Interview Kurverwaltung Bad Birnbach, 2018).

Auf Ebene des Freistaats Bayern übernehmen der Staatssekretär $(S t S)$ und der Staatsminister des Innern (StM) sowie weitere Vertreter des StMI und das StMB eine relevante Rolle für die Legitimation des Projekts und für die Vergabe von Geldern zur Umsetzung des Projekts. Auch wenn diese Instanzen im Gesamtnetzwerk als weniger zentral im Sinne der Zwischenzentralität erscheinen, ist die hybride Funktion der Institutionen und ihrer Repräsentanten als politische Befürworter und verwaltungsrechtliche Koordinatoren unerlässlich für die Definition von Zuständigkeiten und öffentliche Repräsentation der politischen Unterstützung. Sie stellen keine aktiven projektbegleitenden Partner dar, sondern tauchen zeitlich nur vergleichsweise kurz mit hohen Zwischenzentralitätswerten (vgl. Abb. 8.2) im Netzwerk auf, sind aber von erheblicher Relevanz für die politische, verwalterische und gesellschaftliche Befürwortung und Legitimation.

Der TÜV Süd ist letztlich die prüfende Instanz im Bereich des Zulassungsprozesses des Fahrzeugs. Diese Institution hat wesentlichen Einfluss auf den Abgleich der Potenziale der neuen Technologien mit den rechtlichen Rahmenwerken und Bestimmungen. Indirekt werden darüber auch ethische, moralische und gesellschaftliche Fragen ausgehandelt. Der TÜV Süd nimmt eine zentrale Rolle im Hinblick auf die Einhaltung von gesellschaftlich definierten und über lange Zeit ausgehandelten Sicherheitsstandards ein (Kap. 10, Wuth und Dorner). Die dort stattfindenden Lernprozesse und die Erteilung von Ausnahmegenehmigungen können weitreichenden Einfluss auf die gesellschaftliche Legitimation von Neuerungen und Innovationen hinsichtlich ihrer Sicherheit und Zuverlässigkeit haben. Indirekt können durch die genauen Prüfungen und Lernprozesse Grundlagen für neue rechtliche Rahmenwerke geschaffen werden. Dabei sollte nicht unterschätzt werden, dass veränderte deutsche und europäische Regelungen auch Einfluss auf internationale Regelungen und Standards nehmen können.

Auf Bundesebene spielen vor allem politische und wirtschaftliche Repräsentanten eine wichtige Rolle. Beispielsweise wird durch die Bekanntgabe der Durchführung des Pilotprojekts in Bad Birnbach durch die DB-Konzernspitze im Rahmen einer Pressekonferenz in Berlin Ende des Jahres 2016 eine Teilfinanzierung sowie die Koordination des Projekts indirekt zugesagt.

Durch die Beteiligung von Repräsentanten aus Bad Birnbach an einem Beirat des BMVI kann das Pilotprojekt in Bad Birnbach Einfluss auf bundesweite Entwicklungen hinsichtlich Neuerungen und Innovationen haben. Der einzige Akteur, der laut Aussage einiger In- 
terviewpartner das Pilotprojekt zusätzlich hätte legitimieren und unterstützen können, wäre der Bundesminister für Verkehr und Infrastruktur (Bundesminister für VI) gewesen.

Auf transnationaler Ebene ist vor allem das Unternehmen EasyMile zu nennen. EasyMile ist ein französisches Unternehmen, das die Software für automatisierte Fahrzeugsysteme entwickelt. Gemeinsam mit dem französischen Fahrzeughersteller Ligier haben sie den Shuttlebus EZ10 entwickelt. Etwas irreführend ist die Darstellung von EasyMile im Gesamtnetzwerk hinsichtlich der Größe, trotz vergleichsweise wenigen projektrelevanten Verbindungen. Dafür hat EasyMile wichtige Verbindungen zu Investoren (z. B. Continental und Alstom) und Fahrzeugherstellern (z. B. Ligier) und verfügt in diesem Sinne über eine hohe Zwischenzentralität. Ohne Unternehmen wie EasyMile, könnte es Projekte wie in Bad Birnbach nicht geben. Weltweit gibt es Anfang des Jahres 2019 offiziell nur drei Hersteller für automatisierte Shuttlebussysteme (EasyMile, Local Motors, Navya).

Darüber hinaus ist die Presse- und Öffentlichkeitsarbeit - wenngleich mit geringer Zwischenzentralität dargestellt - zum Teil als transnational und international zu verstehen. Die Berichterstattung erfolgt teils in englischer Sprache und wird über digitale Medien verbreitet. Zudem sind die Erzählungen und Bewertungen von internationalen Touristen, die mit dem Shuttlebus in Bad Birnbach gefahren sind, nicht unerheblich für eine internationale Repräsentation, sodass das Projekt potenziellen Einfluss auf die internationale Wahrnehmung autonomer und (teil-)automatisierter Fahrzeugsysteme haben kann. Auch Delegationen aus Politik und Wirtschaft, die sich für das Projekt in Bad Birnbach interessieren und sich über eigene Anwendungsmöglichkeiten in anderen Kontexten und Orten informieren möchten, kommen nicht nur aus Deutschland, sondern teils aus ganz Europa und sind daher als transnational einzustufen.

\subsubsection{Phasenmodell und Akteur-Netzwerke}

Für eine differenzierte und dynamische Analyse der Relevanz der einzelnen Einbettungsdimensionen wurden die stattfindenden Prozesse in Projektphasen eingeteilt und skizziert. Auf dieser Grundlage wurden Akteur-Netzwerke für die definierten drei Projektphasen erstellt. Darüber können unterschiedliche Relevanzen einzelner Akteure, im Sinne ihrer Zwischenzentralität, in den jeweiligen Phasen, und die Entstehungsdynamiken des Netzwerks rekonstruiert werden. Für eine bessere Übersicht sind die Projektphasen, die dominanten Akteure und die identifizierten Einbettungsdimensionen in Tab. 8.1 dargestellt. Anschließend werden die sozialen Netzwerke der einzelnen Phasen abgebildet und detailliert beschrieben (Abb. 8.2).

\section{Initialphase: Anfang 2015 bis Januar 2017 (Abb. 8.2)}

Als Initiatoren des Pilotprojekts in Bad Birnbach werden Vertreter der DB und ihre Tochtergesellschaften Südostbayernbahn ( $S O B$ ) und $R B O$ gesehen. Bereits gegen Anfang des Jahres 2015 (Interview Kurverwaltung Bad Birnbach, 2018) sind Vertreter der DB, bzw. des bis Dezember 2018 mehrheitlich von der DB finanzierten InnoZ in Berlin, während 
Tab. 8.1 Zusammenfassung der Projektphasen und Charakteristika. (Quelle: eigene Darstellung, Leitfadeninterviews, 2018)

Initialphase: Anfang 2015 bis Januar 2017

„Meilensteine“

- ca. Anfang 2015: erster informeller Kontakt zwischen Vertretern aus Bad Birnbach, InnoZ-

Vertreter, Vertretern des LRA Rottal-Inn

- 09/16: erste offizielle Einladung von BaBi-Vertretern durch die DB nach Leipzig (DB-

Schenker-Gelände)

- 10/16: Beginn Probelauf in Leipzig

- 12/16: Pressekonferenz in Berlin: DB-Konzernspitze gibt Pilotprojekt in Bad Birnbach bekannt

Akteure

- InnoZ, DB-Konzernspitze, Vertreter von Bad Birnbach, kommunale Gremien Bad Birnbach, LRA Rottal-Inn

Einbettungsdimensionen und -prozesse

- Einbettungsprozesse in (interne) Organisations- und Unternehmensumfelder (Marktgemeinde Bad Birnbach und DB)

Vorbereitungsphase: Februar 2017 bis September 2017

„Meilensteine“

- ab 02/17: Workshops mit Schlüsselakteuren, gemeinsames Lernen, kommunale

Öffentlichkeitsarbeit und überregionale Kontaktanbahnung (politische Unterstützung generieren)

- 04/17 Erste öffentliche Projektpräsentation in Bad Birnbach

- 04-08/17: Zulassungsprozesse Fahrzeug, Strecke und Linienverkehr

- 04-09/17: Personalakquise und Organisation des Fahrbetriebs

Akteure

- Verbleibende Akteure: Vertreter von Bad Birnbach, kommunale Gremien Bad Birnbach, LRA

Rottal-Inn

- Neue Akteure: Öffentlichkeitsarbeit, Akteure aus Politik und Verwaltung auf Bundeslandebene

(z. B. Landtagsabgeordnete des Stimmkreises Rottal-Inn, Regierungen von Niederbayern und der

Oberpfalz), TÜV Süd, ioki, EasyMile

Einbettungsdimensionen und -prozesse

- Beginnende und rasch voranschreitende Einbettung in Regelungsumfeld und breitere (über) regionale Öffentlichkeit

- Vertiefung der Einbettungsprozesse in interne Organisations- und Unternehmensumfelder

Durchführungsphase: Oktober 2017 bis Dezember 2018

„Meilensteine“

- 10/17: Offizielle Inbetriebnahme mit Pressekonferenz in Bad Birnbach; Regelbetrieb

- 01/18-01/19: Begleitforschung

Akteure

- Verbleibende Akteure: Vertreter von Bad Birnbach, kommunale Gremien Bad Birnbach, LRA

Rottal-Inn, Öffentlichkeitsarbeit, TÜV Süd, ioki, EasyMile

- Neue Akteure: Operator, Nutzer und Nicht-Nutzer, Begleitforschung, Delegationen, Beirat des

BMVI, (trans-)nationale Öffentlichkeitsarbeit 
Tab. 8.1 (Fortsetzung)

Einbettungsdimensionen und -prozesse

- Beginnende Einbettungsprozesse in Nutzerumfelder

- Dadurch auch Vertiefung der Einbettung in breitere Öffentlichkeit und Organisations- und Unternehmensumfelder

- Beginnende transnationale Einbettung durch Öffentlichkeitsarbeit und Diskussion des Wiener Abkommens über den Straßenverkehr

der Suche nach geeigneten Standorten für die Durchführung eines Pilotprojekts zu ,autonom fahrenden Kleinbusse im ÖPNV“ mit einer informellen Anfrage im Rahmen einer Veranstaltung auf einen Vertreter der Gemeinde Bad Birnbach zugegangen. In der Folge wurde diese Idee erstmals zwischen dem Leiter der Kurverwaltung ( $K u r V W B a B i)$, den Bürgermeistern von Bad Birnbach ( $\mathrm{BM} \mathrm{BaBi)}$ und dem Leiter der Kreisentwicklung des Landratsamts Rottal-Inn (KEW Ro-I) informell kommuniziert. Als wichtiger Multiplikator ist also der Kurdirektor und Verantwortliche für die Pressearbeit der Gemeinde Bad Birnbach anzusehen, der die anfängliche Anfrage an die Bürgermeister sowie an Vertreter des Landratsamt Rottal-Inn (v. a. Leiter der Kreisentwicklung) weitergeleitet hat. Alle Akteure zeigten sich begeistert und sehr interessiert, u. a. weil das Thema ÖPNV im ländlichen Raum und besonders im Landkreis Rottal-Inn eine große Herausforderung darstellt. Grund ist, dass der Landkreis einer der streusiedlungsreichsten in Deutschland ist und mit nur „120.000 Einwohnern über weit mehr Haltestellen als München verfügt“ (Interview Kreisentwicklung Rottal-Inn, 2018). Dadurch wird die Bereitstellung von ÖPNV zu einer groBen logistischen und finanziellen Herausforderung für die Verwaltungsinstanzen. Neuen Mobilitätslösungen für den ÖPNV steht man demnach offen gegenüber. In Bezug auf gesellschaftliche Einbettungsprozesse gab es weder auf kommunaler noch auf LandkreisEbene Hürden hinsichtlich der grundsätzlichen Bereitschaft zur Integration in das organisatorische oder institutionelle Umfeld.

Nach einer „Zeit des Wartens“ (Interview Kurverwaltung Bad Birnbach, 2018) erfolgte im September 2016 die erste formelle Einladung von Repräsentanten aus Bad Birnbach zu einem in Leipzig auf dem DB-Schenker-Gelände stattfindenden Projekt zu autonom fahrenden Shuttlebussen, das ab Oktober 2016 durchgeführt wurde. Damit erfolgte der erste formelle Schritt, der zur Umsetzung des Pilotprojekts in Bad Birnbach führte.

Im Dezember 2016 kündigte DB-Chef Rüdiger Grube (DB-Konzernspitze) auf einer Pressekonferenz zu einem Testbetrieb auf dem Gelände des InnoZ in Berlin das ,Pilotprojekt autonom fahrender Kleinbus im ÖPNV in Bad Birnbach“ an (Interview RBO, 2018). Das hat mehrere Funktionen und trägt zu einer Einbettung in die Organisationsstrukturen der Gemeinde Bad Birnbach, des Unternehmens DB und der breiten Öffentlichkeit durch die Medienpräsenz bei. Auf kommunaler und regionaler Ebene wurden Prozesse der strukturellen Einbettung in ein Organisationsumfeld (z. B. Infrastrukturen, Zuständigkeiten auf kommunaler Ebene) angeschoben. Beispielsweise fand die erste Gemeinderatssitzung statt, in der das mögliche Projekt thematisiert und in kommunalen Gremien kommuniziert wurde. 
Zwischen Oktober 2016 und Januar 2017 standen vor allem die interne Kommunikation in Bad Birnbach und innerhalb der DB und ihrer Tochterunternehmen (z. B. Projektkoordination vor Ort) sowie die Kontaktanbahnung und Vereinbarung zur Projektdurchführung in Bad Birnbach im Vordergrund.

Einbettungsprozesse finden in dieser Phase also eher intern im Rahmen des Organisationsumfelds der Gemeinde Bad Birnbach und des Landkreises Rottal-Inn statt. Aber auch innerhalb der DB müssen interne Strukturen für ein solches Projekt geschaffen werden. Grundlegende Zuständigkeiten und Möglichkeiten müssen vor einer Einbettung in eine breitere Gesellschaft oder in ein Nutzerumfeld intern geschaffen werden.

\section{Vorbereitungsphase: Februar 2017 bis Oktober 2017 (Abb. 8.2)}

In der Vorbereitungsphase standen, neben Vertretern der $R B O$ und dem Hersteller EasyMile, die institutionellen Akteure und die Zulassungsverfahren im Mittelpunkt (Regierung von Niederbayern - Reg NBay, Regierung der Oberpfalz - Reg Opf, StMI, StMB, TÜV Süd). Gerade die RegNBay verfügt in dieser Phase über eine auffallend hohe Zwischenzentralität, die weder davor noch danach gegeben ist (Abb. 8.2). Ab Februar 2017 entstanden Arbeitsgruppen aus Vertretern der RBO, unterschiedlicher Gremien von Bad Birnbach, EasyMile, des Landratsamtes Rottal-Inn, TÜV Süds und Delegationen der zuständigen Ministerien (StMI, StMB), die in gemeinsamen Workshops und Treffen Erfahrungen und Wissen generierten. Auf dieser Grundlage konnten Strukturen, Abläufe und Herausforderungen auch auf (über)regionaler Ebene festgelegt werden.

Während in der Phase davor die interne Kommunikation eine wichtige Rolle spielte, ist ab Februar 2017 die Kommunikation nach außen, also mit Institutionen auf Landes- und Bundesebene, aber auch mit zivilgesellschaftlichen Akteuren auf kommunaler Ebene ( $\mathrm{An}$ wohner) dominierend. Es lassen sich erste Einbettungsprozesse in eine breitere Öffentlichkeit auf kommunaler Ebene ( $\ddot{O A} k$ ) identifizieren. Vor einer offiziellen Projektdurchführung ist es ratsam, das Projekt bereits von der kommunalen Öffentlichkeit legitimieren zu lassen. Dies kann durch Informationsveranstaltungen für Anwohner oder spezifische Zielgruppen (z. B. Gastronomen, Landwirte, Schüler) vorangetrieben werden. Auch kommunale Gremien (z. B. der Gemeinderat) können solche Vorhaben vorantreiben oder blockieren.

In dieser Phase kann nicht nur von vertiefender Einbettung in das Organisationsumfeld gesprochen werden, sondern auch von Einbettungsprozessen in das Regelungsumfeld und in die breitere regionale und überregionale Gesellschaft. Ziel war es, auf allen gesellschaftlichen und politischen Ebenen, Zustimmung zu erzeugen und so auch die Finanzierbarkeit des Projekts sicherzustellen. So wirkt sich die gesellschaftliche Legitimation offensichtlich auch auf die Finanzierbarkeit aus.

Wiederholt betont wurde die Rolle der Abgeordneten des Bayerischen Landtags für den Stimmkreis Rottal-Inn (MdLSK Ro-I). Sie nahm die Rolle als „Politische Botschafterin fürs Geld“ ein (Interview Abgeordnete Stimmkreis Rottal-Inn, 2018) und stellte die Verbindung zu politischen Akteuren und Institutionen auf Bundes- und Landesebene her (z. B. Bayerischer Staatsminister (StM) und Staatssekretär (StS) im StMI), ohne deren Befürwortung eine Durchführung auf Verwaltungsebene nicht legitimierbar und für die Kom- 
mune nicht finanzierbar gewesen wäre. Ihre zentrale Rolle während dieser Projektphase wird auch durch das hohe Maß an Zwischenzentralität im Netzwerk bestätigt (Abb. 8.2). Erst durch die politische Befürwortung konnten Zuständigkeiten, bspw. im Rahmen des Zulassungsprozesses, definiert werden und die Sonderzulassungsverfahren durchgeführt werden.

Der Zulassungsprozess dauerte von April bis August 2017. Dabei erfolgte in einem ersten Schritt eine Vorankündigung für eine Antragsstellung für eine Liniengenehmigung im ÖPNV bei der Regierung von Niederbayern. Voraussetzungen für eine solche Zulassung sind ein zugelassenes Fahrzeug und eine klar definierte Route. Sowohl der automatisierte Kleinbus EZ10 bedurfte einer Sondergenehmigung durch die Regierung der Oberpfalz (bayernweite Zuständigkeit für Sonderzulassungsverfahren), als auch die Strecke, die durch die Behörden in Sonderzulassungsverfahren bearbeitet werden musste. Die eigentliche Fahrzeugzulassung erfolgte mittels der Sondergenehmigung beim Landratsamt Rottal-Inn. Diese Fahrzeugzulassung mit Sondergutachten konnte gemeinsam mit der Streckengenehmigung bei der Regierung von Niederbayern vorgelegt werden, um eine Konzession für eine ÖPNV-Linie zu beantragen (Interview RBO, 2018).

Geprägt war diese Phase von den Herausforderungen, ein bis dato unbekanntes Produkt/Objekt zuzulassen, Abläufe und Prozesse erstmals durchzuführen und Zuständigkeiten zu definieren. Von Hersteller-Seite mussten mehrfach Anpassungen am Produkt vorgenommen werden. Es kam zu diversen Kommunikations- und Interaktionsprozessen, die gekennzeichnet sind durch Lernprozesse und Feedbackschleifen zwischen den unterschiedlichen Akteuren und auch als Co-Konstruktion von Technologie und Umfeld bezeichnet werden können. An der Komplexität des Vorgangs wird deutlich, dass entsprechender unternehmerischer und politscher Wille dahinterstecken muss, um einen solchen Zulassungsprozess durchzuführen.

Ein Schlüsselereignis stellt die erste Projektpräsentation des ,,autonom fahrenden Kleinbusses im ÖPNV“ in Bad Birnbach im April 2017 dar, während der der Bus erstmals in Bad Birnbach vorgeführt wurde und so auch mediale Präsenz erzeugte. „Wenn man sich sicher ist, dass man das irgendwie hinkriegt, dann muss das gleich mal raus an die Öffentlichkeit. Weil, dann finden sie keinen mehr, der offiziell sagen kann, nein das geht nicht" (Interview Kreisentwicklung Rottal-Inn, 2018). Mediale Präsenz hat hier einen gesellschaftlichen Einbettungsprozess unterstützt. Dadurch konnte sich auch die Einbettung in ein Regelungsumfeld verstärken bzw. erst möglich gemacht werden. Durch die offizielle Ankündigung entstand auf diversen Ebenen Handlungsdruck, der dazu beitrug, der öffentlichen Verwaltung die Handlungsmacht zu verleihen, ein bislang unbekanntes Produkt zuzulassen.

Betont wurde in mehreren Interviews, dass das für eine Verwaltung unkonventionelle und beherzte Vorgehen der Gemeinde Bad Birnbach und des Landratsamtes für die schnelle Umsetzung des Projekts in Bad Birnbach förderlich waren (z. B. Interview RBO, 2018; Interview Bürgermeister, 2018; Interview Regierung Niederbayern, 2018). Dazu beigetragen hat auch die Organisationsstruktur der Kreisentwicklung des Landkreises Rottal-Inn, charakterisiert durch kurze Wege und engen inhaltlichen Austausch zwischen den einzelnen Ressorts. Hervorzuheben ist die hybride Rolle des Leiters der Kreisentwicklung, der 
Erfahrungen und Wissen aus Arbeit in der freien Wirtschaft und in der öffentlichen Verwaltung vereinen kann.

Zwischen April und September 2017 konnten zudem die gesamte Personalakquise (z. B. Operator), die Organisation des Fahrbetriebs, der Unterstellung und der Batteriebetankung abgeschlossen werden. Das heißt, neben der Einbettung in das Regelungsumfeld konnte die Einbettung in das Organisationsumfeld vertieft werden.

\section{Durchführungsphase: Oktober 2017 - Januar 2019 (Abb. 8.2)}

Von Oktober 2017, als die offizielle Inbetriebnahme mit Pressekonferenz in Bad Birnbach stattfand, bis heute kann von der Durchführungsphase gesprochen werden. Als neue Akteure sind die durchführenden und beobachtenden Akteure zu nennen. Dazu gehören die Nutzer und Nicht-Nutzer, Anwohner und die Operatoren ebenso wie die Akteure der Begleitforschung, die hier nicht dargestellt sind (Abschn. 8.4.1). Die Operatoren verfügen an dieser Stelle über eine vergleichsweise hohe Zwischenzentralität und wurden auch im Rahmen der Begleitforschung als wichtige Beobachter und Mediatoren befragt (Kap. 5, Kolb et al.), da sie zwischen Nutzern, Marktgemeinde und Unternehmen interagieren.

In koordinierender Funktion verbleiben $R B O$ und ioki als zentrale Akteure ebenso wie EasyMile, die in permanentem Austausch stehen und ihr Produkt entsprechend verbessern und anpassen. Zeitgleich laufen ähnliche Prozesse wie während der Vorbereitungsphase ab, beispielsweise im Hinblick auf den Zulassungsprozess für ein zweites Fahrzeug (Februar 2018) und die Streckenerweiterung (Interview RBO, 2018). Die Kommunikation nach außen nimmt stetig zu und zeigt sich auch in einer zunehmenden Anzahl von Delegationen (z. B. anderer Gemeinden, Unternehmen, politischer Vertreter) und Besuchern, die sich für den Bus interessieren, aber ebenso in der Mitwirkung von Vertretern der Gemeinde Bad Birnbach in einem Beirat des Verkehrsministeriums (Beirat BMVI) auf Bundesebene. Auch diese Mitarbeit kann Einfluss auf bundesweite oder europaweite Ausarbeitung von Regelungen haben. Das seit Jahrzehnten als Grundlage dienende Wiener Übereinkommen über den Straßenverkehr wird mit diesen Sondergenehmigungen in Frage gestellt. Grundsätzlich kann das Auswirkungen auf eine europäische Gesetzgebung haben und so einen Hinweis auf transnationale Einbettungsprozesse darstellen.

Insgesamt ist diese Phase charakterisiert durch Maßnahmen zur Steigerung der Akzeptanz bei Bevölkerung und Nutzern. Öffentlichkeitsarbeit, Pressetermine, aber auch die Verstetigung getesteter Abläufe und das Generieren von Zuverlässigkeit (z. B. Fahrpläne einhalten, Anbindung des Bahnhofs) tragen zu einer beginnenden gesellschaftlichen Einbettung in Nutzerumfelder und die Gesellschaft im weiteren Sinne bei. Zu letzterem gehören Akteure aus sämtlichen gesellschaftlichen Teilbereichen - sowohl aus Zivilgesellschaft, Politik oder Verwaltung, als auch aus Wirtschaft, Verbänden oder Interessensvertretungen.

In den Hintergrund treten dafür die institutionellen Akteure - vor allem auf Landesebene. Daran wird deutlich, dass diese Akteure zeitlich nur punktuell, aber hochgradig relevante Positionen einnehmen. Ohne deren Unterstützung wäre eine Umsetzung keinesfalls möglich gewesen, da die Einbettung in ein Regelungsumfeld blockiert wäre. 


\subsection{Zusammenfassung und Ausblick}

Am Fallbeispiel des Pilotprojekts in Bad Birnbach können drei charakteristische Projektphasen identifiziert werden, in denen unterschiedliche Aspekte der gesellschaftlichen Einbettung im Vordergrund stehen. Diese Einbettungsdimensionen spiegeln sich auch in den sich über die einzelnen Projektphasen wandelnden Netzwerk- und Akteur-Konfigurationen wider. Während in der Initialphase Prozesse der Einbettung in Unternehmens- und Organisationsumfeld im Vordergrund standen - also Prozesse der internen Einbettung - gewinnen während der Vorbereitungsphase langsam auch Prozesse der externen Einbettung an Bedeutung, allen voran Einbettungsprozesse in eine breitere gesellschaftliche Öffentlichkeit und in ein Regelungsumfeld. Dabei kann durch die Einbettung in eine breite Öffentlichkeit Handlungsdruck zur Umsetzung des in der Öffentlichkeit positiv konnotierten Projekts entstehen und dazu beitragen, die Einbettung in ein Regelungsumfeld zu befürworten. Das gilt auch umgekehrt, die Einbettung in ein Regelungsumfeld, durch Instanzen wie TÜV Süd sowie die Regierungen von Niederbayern und der Oberpfalz, begünstigen das Vertrauen der breiteren Öffentlichkeit und positive Diskurse. Während der Durchführungsphase gewinnen Prozesse der externen Einbettung zunehmend an Dynamik und Reichweite. Dazu gehört die Einbettung in eine breitere gesellschaftliche Öffentlichkeit und in transnationale Umfelder, die durch Öffentlichkeitsarbeit, politische Befürwortung sowie die unmittelbare Erfahrbarkeit der Technologie in Bad Birnbach unterstützt wird. Zeitgleich findet eine zunehmende Verstetigung und Routinisierung der Strukturen und Abläufe in den unternehmens- und organisationsinternen Bereichen statt.

Zusammenfassend lässt sich sagen, dass die Umsetzung des Pilotprojekts in Bad Birnbach nur durch die Befürwortung und Legitimation einer Vielzahl von Akteuren und Mediatoren auf unterschiedlichen Ebenen und aus unterschiedlichen Teilbereichen der Gesellschaft umgesetzt werden konnte. Das bedeutet auch, dass nicht nur die Akzeptanz der Nutzer oder Marktdynamiken über die Umsetzung von Alternativen im Verkehrs- und Mobilitätssektor entscheiden. Besonders die Zustimmung von Akteuren aus Politik und Verwaltung ist wegweisend für die Möglichkeiten alternativer Technologien in diesem Bereich. Gerade die Verbindungen zwischen kommunaler Politik und bayernweiter Landespolitik hatten wesentlichen Einfluss auf die Finanzierbarkeit und die Möglichkeit der Regelung der komplexen Zuständigkeiten. Solche Komplexitäten können auch instrumentalisiert werden, um die Umsetzung von Vorhaben zu erschweren. Dies war aber nicht der Fall im Rahmen des Pilotprojekts in Bad Birnbach. Zudem ist hervorzuheben, dass die Verbindungen zu den relevanten Ministerien auf bayerischer Ebene nur vergleichsweise kurz bestanden, dafür aber von außerordentlicher Relevanz waren. Erst dadurch wurde das Projekt zur Durchführung legitimiert, Zuständigkeiten wurden definiert und die Finanzierung abgesichert. Neben den nur zeitweise als relevant erscheinenden Akteuren gibt es auch einige Akteure, die das Projekt durchgehend begleitet haben und eine wichtige Rolle für die Kontinuität, das Projektmanagement und die Kommunikation nach außen eingenommen haben (u. a. RBO, Kurverwaltung, Kreisentwicklung). Insbesondere die hybriden Akteure, die Erfahrungen und Kontakte in unterschiedlichen gesellschaftlichen Teilbereichen (z. B. 
freie Wirtschaft und Verwaltung) haben, haben die zügige Umsetzung des Projekts vorangetrieben.

Grundsätzlich sind ,,autonom fahrende Kleinbusse“ zwar noch weit entfernt von einer gesellschaftlichen Einbettung, die disruptive Auswirkungen auf bestehende soziotechnische Systeme haben könnte - das wird allein daran deutlich, dass der EZ10 in Bad Birnbach eher als (teil-)automatisiertes Fahrzeugsystem anzusehen ist - dennoch finden weitreichende Lernprozesse mit neuen Produkten und Technologien sowie ihren Wechselwirkungen mit gesellschaftlichen Umfeldern statt. Explizit hervorzuheben sind Lern- und Erfahrungsprozesse im Regelungsumfeld, in der breiteren Öffentlichkeit und im Unternehmerumfeld. Aber auch transnational können gesellschaftliche Einbettungsprozesse automatisierter und autonom fahrender Fahrzeugtechnologien in der medialen Berichterstattung beobachtet werden.

\section{Literatur}

Appel A (2016) Embeddedness and the (re)making of retail space in the realm of multichannel retailing: the case of Migros Sanal Market in Turkey. Geografiska Annaler Series B - Human geography 98/1:55-69

Coe N, Lee Y (2013), We've learned how to be local': the deepening territorial embeddedness of Samsung-Tesco in South Korea. Journals of Economic Geography 13:327-356

Czernek-Marszałek K (2017) Applying mixed methods in social network research - The case of cooperation in a Polish tourist destination. Journal of Destination Marketing \& Management 11:40-52

DB - Deutsche Bahn (2018) Testfeld Bad Birnbach - Erste autonome Buslinie Deutschlands im öffentlichen Straßenraum. URL: https://www.deutschebahn.com/de/Digitalisierung/autonomes_ fahren_neu/Testfeld_Bad_Birnbach-1206846 (20.02.2019)

Deuten JJ, Rip A, Jelsma J (1997) Societal embedding and product creation management, Technology Analysis and Strategic Management 9/2:131-148

EasyMile (2018) Our products. URL: http://www.easymile.com/\#Products (20.02.2019)

Franke K, Wald A (2006) Möglichkeiten der Triangulation quantitativer und qualitativer Methoden in der Netzwerkanalyse, In: Hollstein B, Straus F (Hrsg) Qualitative Netzwerkanalyse, Konzepte, Methoden, Anwendungen. VS Verlag für Sozialwissenschaften, Wiesbaden, S 153-176

Geels FW, Verhees B (2011) Cultural legitimacy and framing struggles in innovation journeys: A cultural-performative perspective and a case study of Dutch nuclear energy (1945-1986). Technological Forecasting \& Societal Change 78:910-930

Granovetter M (1985) Economic action and economic structure: The problem of embeddedness. American Journal of Sociology 91:481-510

Hollstein B (2014) Mixed methods social networks research: An introduction. In: Domínguez S, Hollstein B (Hrsg) Mixed methods social networks research: Design and Applications. Cambridge University Press, New York, S 3-34

Hollstein B (2006) Qualitative Methoden und Netzwerkanalyse - ein Widerspruch? In: Hollstein B, Straus F (Hrsg) Qualitative Netzwerkanalyse, Konzepte, Methoden, Anwendungen. VS Verlag für Sozialwissenschaften, Wiesbaden, S 11-36

Jack S (2010) Approaches to studying networks: Implications and outcomes. Journal of Business Venturing, 25/1:120-137 
Jacobsson S, Lauber V (2006) The politics and policy of energy system transformation: explaining the German diffusion of renewable energy technology. Energy Policy 34/3:256-276

Jansen D, Diaz-Bone R (2014) Netzwerkstrukturen als soziales Kapital. In: Weyer J (Hrsg) Soziale Netzwerke. Konzepte und Methoden der sozialwissenschaftlichen Netzwerkforschung. Oldenbourg Verlag, München, S 71-104

Jansen D (2006) Einführung in die Netzwerkanalyse: Grundlagen, Methoden, Forschungsbeispiele, VS Verlag für Sozialwissenschaften, Wiesbaden

Kanger L, Geels FW, Sovacool B, Schot J (2018) Technological diffusion as a process of societal embedding: Lessons from historical automobile transitions for future electric mobility. Transportation Research Part D: Transport and Environment. doi: https://doi.org/10.1016/j.trd.2018.11.012

Lovell H (2008) Discourse and innovation journeys: the case of low energy housing in the UK. Technol Anal Strateg 20/5:613-632

Mylan J, Morris C, Beech E, Geels FW (2018) Rage against the regime: Niche-regime interactions in the societal embedding of plant-based milk. Environ. Innov. Soc. Trans. doi.https://doi. org/10.1016/j.eist.2018.11.001.

Pike A, Lagendijk A, Vale M (2000) Critical reflections on ,embeddedness' in economic geography: labour market governance in the North East region of England. In: Giunta A, Lagendijk A, Pike A (Hrsg): Restructuring industry and territory: the experience of Europe's regions, TSO, London, S. $59-82$

Polanyi K. (1957) The great Transformation - The Political and Economic Origins of Our Time. Beacon Press, Boston Massachusetts

Schnegg M (2010) Strategien und Strukturen. Herausforderung der qualitativen und quantitativen Netzwerkforschung. In: Gamper M, Reschke L (Hrsg) Knoten und Kanten, Soziale Netzwerkanalyse in Wirtschafts- und Migrationsforschung. Transcript, Bielefeld, S 55-75

Open Access Dieses Kapitel wird unter der Creative Commons Namensnennung 4.0 International Lizenz (http://creativecommons.org/licenses/by/4.0/deed.de) veröffentlicht, welche die Nutzung, Vervielfältigung, Bearbeitung, Verbreitung und Wiedergabe in jeglichem Medium und Format erlaubt, sofern Sie den/die ursprünglichen Autor(en) und die Quelle ordnungsgemäß nennen, einen Link zur Creative Commons Lizenz beifügen und angeben, ob Änderungen vorgenommen wurden.

Die in diesem Kapitel enthaltenen Bilder und sonstiges Drittmaterial unterliegen ebenfalls der genannten Creative Commons Lizenz, sofern sich aus der Abbildungslegende nichts anderes ergibt. Sofern das betreffende Material nicht unter der genannten Creative Commons Lizenz steht und die betreffende Handlung nicht nach gesetzlichen Vorschriften erlaubt ist, ist für die oben aufgeführten Weiterverwendungen des Materials die Einwilligung des jeweiligen Rechteinhabers einzuholen.

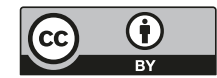

